\title{
Elucidating Direct Kinase Targets of Compound Danshen Dropping Pills Employing Archived Data and Prediction Models
}

Tongxing Wang¹, Lu Liang², Chunlai Zhao ${ }^{1}$, Jia Sun¹, Hairong Wang ${ }^{1}$, Wenjia Wang', Jianping Lin ${ }^{2}$, Yunhui Hu${ }^{1}$

1. GeneNet Pharmaceuticals Co. Ltd., No.1, Tingjiang West Road, Beichen District, Tianjin 300410, China

2. College of Pharmacy, Nankai University, 38 Tongyan Road, Haihe Education Park, Jinnan District, Tianjin 300353, China.

\section{Abstract}

The research on the direct target of traditional Chinese medicine (TCM) is the key to study the mechanism and material basis of TCM, but there is still no effective technical methods at present. For Compound Danshen dropping pills (CDDP), there is no report about its direct targets. In this study, the direct targets of CDDP were studied for the first time, especially focusing on the protein kinase family, which plays causal roles in a variety of human disease. Firstly, the literature database of CDDP was constructed by literature retrieval, and the important components contained in CDDP were extracted. Secondly, the potential direct targets of important components was obtained through querying public database and predicted by Multi-voting SEA algorithm. Then, the KinomeX system was used to predict and to filter the potential kinase targets of CDDP. Finally, the experimental verification was carried out. In total, 30 active kinase targets was obtained at $25 \mu \mathrm{g} / \mathrm{ml}$ concentration of CDDP, and 9 dosedependent targets were obtained at $250 \mu \mathrm{g} / \mathrm{ml}$ concentration of CDDP. This is an efficient and accurate strategy by integrating the targets recorded in several public databases and the targets calculated by two in silico modelling approaches predict potential direct targets of TCM, which can lay an important foundation for the study of the mechanism and material basis of them, promoting the modernization of TCM.

Keywords: Compound Danshen Dropping Pill; Traditional Chinese Medicine; Kinase; Direct target; Allosteric effect; Mechanism of action. 


\section{Introduction}

Traditional Chinese medicine prescriptions are the characteristics of TCM. They have been practiced for thousands of years and have been proved to be effective in modern clinical practice. These prescriptions embodies the dialectical thought of TCM and the medication holistic view. However, there is still a lack of effective approaches to systematically study its mechanism and material basis. In recent years, the reductionist research model has accumulated a lot of data, and also provided illuminating research results, such as the discovery of artemisinin [1]. However, considering the complexity of TCM, it is still difficult to completely separate and identify the effective components through the existed technology and analytical methods, let alone to make clear all the effective components in the TCM. Therefore, the research of reductionism is not capable of answering the essential question of the overall efficacy of TCM. It may lead to deviate from the system theory of TCM, so it needs to be combined with the system theory. In recent years, a variety of "omics" techniques based on system theory have been widely used in the research of TCM [25], which can be used to better understand the pharmacological characteristics of TCM [6-9], but still cannot fully reveal the nature of it. Comprehensively understanding the mechanism of synergism among the effective components, drug targets and metabolic pathways is still highly demanded. One key to solve the problem is to carry out the research on the direct target of TCM. It can not only clarify the pharmacological mechanism of TCM from the origin and scientifically interpret its traditional efficacy, but also unveil new disease mechanism [10] and provide reasonable estimation of TCM repositioning. However, many obstacles cannot be ignored in the study of the direct target of TCM, such as the complex ingredients in it, the complex process of metabolism in vivo, the complicated way playing a role in vivo, and so on. At present, technical methods to screen and determine the direct targets for TCM efficiently and accurately are still poorly developed, which hinders elucidating the mechanisms of TCM essentially.

Compound Danshen dropping pills (CDDP) consist of Radix Salviae (Danshen), 
Panax Notoginseng (Burk.) F. H. Chen Ex C. Chow (Sanqi), Borneolum Syntheticum (Bingpian). It is widely used in the prevention, treatment and emergency treatment of coronary artery disease (CAD) and angina pectoris. Although many research articles about CDDP have been published already, the research on its mechanism of action is still not in-depth [11-14]. Most studies focused on the genes or proteins regulated by CDDP treatment, most of which can be referred as indirect targets, but there is no report on the direct targets of CDDP.

At present, kinases belong to an important class of drug targets. Among them, protein kinases family is the largest group of kinases, which act on specific proteins and change their activities. These kinases play a wide range of roles in cell signaling and complex life activities, and their dysfunction plays an important causal role in many human diseases, including cancer, inflammatory diseases, central nervous system diseases, cardiovascular diseases and so on [15].

In view of the importance of kinases, we proposes a new systematic approach to explore direct kinase targets of TCM efficiently and reliably, CDDP was taken as the research subject. This strategy is based on the known activity data recorded in free databases and predicted data calculated by computational models, which is independent of any specific disease model. Firstly, the literature database of CDDP was constructed by literature retrieval, and the important components contained in CDDP were extracted. Secondly, the potential targets of important components was obtained through public database querying and Multi-voting SEA algorithm predicting. Then, the KinomeX system was used to filter the potential kinase targets of CDDP. Finally, 30 active targets were obtained (Figure 1). The activity of MET, PIM1 and SYK had obvious dosedependent effect, and that of CAMK2G, CSF1R, FYN and RET increased at high concentration, which are worthy of further study. 


\section{Methods}
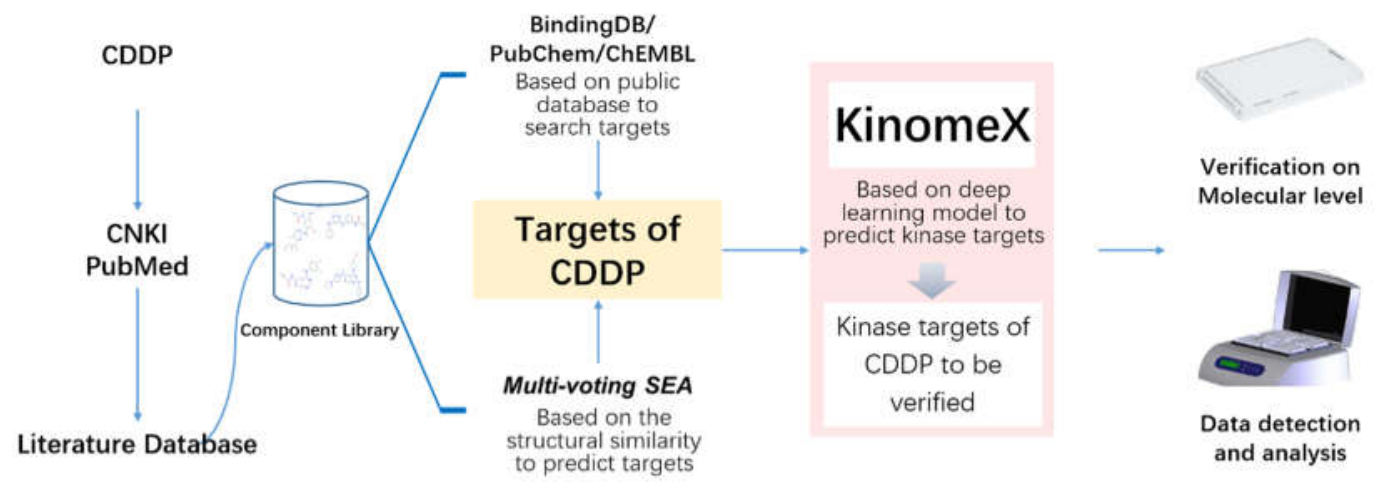

Obtain the important components of CDDP

The prediction of kinase targets for CDDP

Experimental verification

Figure 1. The flowchart of the study based on known activity data and prediction model to obtain the direct kinase targets of CDDP

\section{Construction of important component set for CDDP}

In order to review the literatures related to CDDP as comprehensively as possible, we firstly customized the relevant search terms of it. The Chinese literature retrieval word was "Danshen Dropping Pills", and the English literature retrieval words were "Compound Danshen dripping pills", "Fufang Danshen Diwan", "T89", "Dantonic" and "Cardiotonic Pills". Secondly, we used "Danshen Dropping Pills" as the keyword to obtain the Chinese-language literatures through CNKI (https://www.cnki.net/). Similarly, through PubMed (https://pubmed.ncbi.nlm.nih.gov/), "Compound Danshen drilling pills", "Fufang Danshen Diwan", "T89", "dantonic" and "Cardiotonic Pills" were used to get the English-language literatures (time to December 15, 2019). Finally, the literature database of CDDP was constructed by combining the above literatures from two sources.

The components contained in CDDP were extracted manually by reading the literature, and then the information of them was standardized through PubChem database (https://pubchem.ncbi.nlm.nih.gov/) [16]. It is generally believed that the ingredients entering the blood, main metabolites, bioequivalence components 
compared to the prescription, and active components of CDDP reported in literatures are the important components in CDDP. Additionally, in order to avoid missing critical components included in CDDP, we selected the most extensively studied component in the three single herbs but still unconfirmed in the whole prescription, through retrieving TCM related databases, such as TcmSP [17]、TCMID [18]、TCM-ID [19]、ETCM [20]、YaTCM [21].

\section{Prediction of kinases targets of CDDP}

The known activity data of 40 important components in CDDP were obtained from three authoritative public databases, namely, ChEMBL [22], PubChem [16], BingdingDB [23]. The kinase targets with definite activity information were standardized and screened.

Avoiding missing some important potential kinase targets, Multi-voting SEA algorithm [24] was utilized to predict potential direct targets of important components. In this algorithm, four scoring models, namely Topological SEA, Morgan SEA, MACCS SEA Atom Pair SEA, were integrated to calculate potential targets of components. Finally, all potential targets of each component were normalized and the kinase targets were selected.

The KinomeX system (https://kinome.dddc.ac.cn/en/) [25] developed by Shanghai Pharmaceutical Institute was used to predict the potential kinase targets of 40 important components of CDDP. It enables users to predict its potential kinase targets based on the structure of a given molecule. The prediction results were used to screen the targets obtained from public databases and Multi-voting SEA algorithm mentioned above. The kinase targets screened by results from KinomeX were subsequently taken as the kinase target set of CDDP with high reliability to conduct following experimental verification.

\section{Experimental verification for direct targets of CDDP}

Full KP panel [km ATP], a kinaseprofiler, was developed by Eurofins company. In 
this study, we used this panel to carry out experimental verification for direct kinase targets of CDDP. Firstly, the filter binding radioactive kinase activity assays were performed by using $25 \mu \mathrm{g} / \mathrm{ml}$ of CDDP. The kinase activity inhibition rate of the sample were expressed as the percentage of the result of sample compared to the blank group. The kinase activity of the blank was considered to be $100 \%$. Generally speaking, if the residual enzyme activity is less than $30 \%$, it is considered to be strongly inhibited. And if the residual enzyme activity is between $30 \%$ and $70 \%$, it is considered as moderate inhibition. Considering the weak interaction superposition characteristic and synergistic effect of TCM ingredients [26, 27], the threshold value in this study was set to $80 \%$.

In order to get the dose-dependent kinase targets, the kinase targets with activity value less than 70 were retested at $250 \mu \mathrm{g} / \mathrm{ml}$ of CDDP. The targets with obvious dose dependence characteristic were selected to get the IC50 curve through multiconcentration test. 


\section{Results}

\section{Important components in CDDP}

3719 Chinese-language literatures and 59 English-language literatures were obtained through retrieving the customized terms of the CDDP (time to December 15, 2019). Through literature reading manually, the components information of CDDP was extracted. According to the screening criteria of important ingredients, a total of 39 ingredients were collected. In addition, quercetin, a potential important component of the whole prescription was also included for subsequent analysis, which was reported a lot in single herbs but has not been confirmed in the whole prescription. All 40 important components of CDDP are shown in Table 1.

Table 1. 40 important components of CDDP

\begin{tabular}{|c|c|c|c|}
\hline \# & Component name & Standard InChI Key & PubChem CID \\
\hline CDDP 01 & Danshensu & PAFLSMZLRSPALU-MRVPVSSYSA-N & 11600642 \\
\hline CDDP 02 & Tanshinone I & AIGAZQPHXLWMOJ-UHFFFAOYSA-N & 114917 \\
\hline CDDP 03 & Cryptotanshinone & GVKKJJOMQCNPGB-JTQLQIEISA-N & 160254 \\
\hline CDDP 04 & Tanshinone IIA & HYXITZLLTYIPOF-UHFFFAOYSA-N & 164676 \\
\hline CDDP 05 & Dihydrotanshinone I & HARGZZNYNSYSGJ-JTQLQIEISA-N & 11425923 \\
\hline CDDP 06 & Salvianolic acid A & YMGFTDKNIWPMGF-UCPJVGPRSA-N & 5281793 \\
\hline CDDP 07 & Salvianolic acid B & SNKFFCBZYFGCQN-PDVBOLEISA-N & 11629084 \\
\hline CDDP 08 & Salvianolic acid B & SNKFFCBZYFGCQN-ZAEDQPKISA-N & 124518070 \\
\hline CDDP 09 & Salvianolic acid D & KFCMFABBVSIHTB-WUTVXBCWSA-N & 75412558 \\
\hline CDDP 10 & Salvianolic acid G & KFCMFABBVSIHTB-ZZXKWVIFSA-N & 11683160 \\
\hline CDDP 11 & Protocatechuic aldehyde & IBGBGRVKPALMCQ-UHFFFAOYSA-N & 8768 \\
\hline CDDP 12 & Rosmarinic acid & DOUMFZQKYFQNTF-WUTVXBCWSA-N & 5281792 \\
\hline CDDP 13 & Lithospermic acid & UJZQBMQZMKFSRV-RGKBJLTCSA-N & 6441498 \\
\hline CDDP 14 & Ginsenoside-Rg1 & YURJSTAIMNSZAE-HHNZYBFYSA-N & 441923 \\
\hline CDDP 15 & Ginsenoside-Rb1 & GZYPWOGIYAIIPV-JBDTYSNRSA-N & 9898279 \\
\hline CDDP 16 & Ginsenoside-Rh1 & RAQNTCRNSXYLAH-RFCGZQMISA-N & 12855920 \\
\hline CDDP 17 & Ginsenoside-Rd & RLDVZILFNVRJTL-IWFVLDDISA-N & 11679800 \\
\hline CDDP 18 & Notoginsenoside R1 & LLPWNQMSUYAGQI-OOSPGMBYSA-N & 441934 \\
\hline CDDP 19 & Ginsenoside Re & PWAOOJDMFUQOKB-WCZZMFLVSA-N & 441921 \\
\hline CDDP 20 & Borneol & DTGKSKDOIYIVQL-QXFUBDJGSA-N & 1201518 \\
\hline CDDP 21 & Isoborneol & DTGKSKDOIYIVQL-OYNCUSHFSA-N & 6973640 \\
\hline CDDP 22 & Caffeic acid & QAIPRVGONGVQAS-DUXPYHPUSA-N & 689043 \\
\hline CDDP 23 & Tanshinone IIB & XDUXBBDRILEIEZ-LJQANCHMSA-N & 9926694 \\
\hline CDDP 24 & Methylenetanshinquinone & QDFFAXSMLUUJSG-UHFFFAOYSA-N & 105118 \\
\hline CDDP 25 & Salvianolic acid C & GCJWPRRNLSHTRY-VURDRKPISA-N & 13991590 \\
\hline
\end{tabular}




\begin{tabular}{lccc}
\hline CDDP 26 & Ginsenoside-Rf & UZIOUZHBUYLDHW-XUBRWZAZSA-N & 441922 \\
CDDP 27 & Ginsenoside-F2 & SWIROVJVGRGSPO-JBVRGBGGSA-N & 9918692 \\
CDDP 28 & Ginsenoside-F1 & XNGXWSFSJIQMNC-FIYORUNESA-N & 9809542 \\
CDDP 29 & Ginsenoside-Rb2 & NODILNFGTFIURN-GZPRDHCNSA-N & 6917976 \\
CDDP 30 & Ginsenoside-Rb3 & NODILNFGTFIURN-USYOXQFSSA-N & 12912363 \\
CDDP 31 & Ginsenoside-Rg2 & AGBCLJAHARWNLA-DQUQINEDSA-N & 21599924 \\
CDDP 32 & Notoginsenoside-R2 & FNIRVWPHRMMRQI-PGOMJGFXSA-N & 21599925 \\
CDDP 33 & 20(S)-Ginsenoside Rg3 & RWXIFXNRCLMQCD-JBVRGBGGSA-N & 9918693 \\
CDDP 34 & 20(R)-Ginsenoside Rg3 & RWXIFXNRCLMQCD-CZIWJLDFSA-N & 46887680 \\
CDDP 35 & Miltirone & FEFAIBOZOKSLJR-UHFFFAOYSA-N & 160142 \\
CDDP 36 & protocatechuic acid & YQUVCSBJEUQKSH-UHFFFAOYSA-N & 72 \\
CDDP 37 & catechol & YCIMNLLNPGFGHC-UHFFFAOYSA-N & 289 \\
CDDP 38 & vanillic acid & WKOLLVMJNQIZCI-UHFFFAOYSA-N & 8468 \\
CDDP 39 & 4-hydroxy-3- & SVYIZYRTOYHQRE-UHFFFAOYSA-N & 160637 \\
& methyloxyphenyl lactic acid & & \\
CDDP 40 & quercetin & REFJWTPEDVJJIY-UHFFFAOYSA-N & 5280343 \\
\hline
\end{tabular}

\section{The potential kinase targets of CDDP}

Based on the hypothesis that the potential direct targets of important components in the whole prescription are more likely to be direct targets of the whole prescription we carried out in the following analysis. Therefore, the potential targets of components were used to speculate the potential direct targets of the whole prescription. Three authoritative public databases and Multi-voting SEA algorithm were utilized to obtain direct targets information of important components. Moreover, the KinomeX system was used to predict the kinase targets, and finally the whole set of kinase targets of CDDP was obtained. Through querying the three public databases, 262 known targets including 55 kinase targets (Figure. 2, Table S1) were obtained, and 377 targets including 121 kinase targets (Figure. 2, Table S2) were predicted based on the Multivoting SEA algorithm. By integrating the above two parts of targets, a total of 479 potential direct targets were obtained, including 148 kinase targets (Table 2, Table S3).

Table 2. Statistics of the number of potential direct targets for 40 important components of

CDDP

\begin{tabular}{ccc}
\hline Target sources & Number of targets & Number of kinase targets \\
\hline Recorded targets & 262 & 55 \\
Multi-Voting SEA & 377 & 121 \\
predicted targets & & \\
\hline
\end{tabular}




\begin{tabular}{ccc}
\hline Common targets & 160 & 28 \\
Total targets & 479 & 148 \\
\hline
\end{tabular}

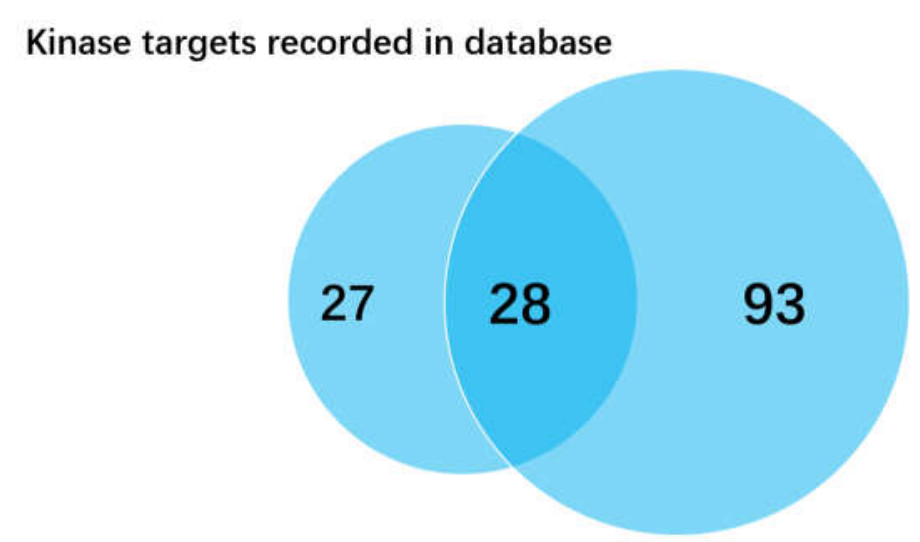

Kinase targets predicted by Multi-Voting SEA

Figure 2. The number of potential direct kinase targets of CDDP

KinomeX, established by the research team of Shanghai Institute of pharmaceutical research, Chinese Academy of Sciences in 2019, is a prediction and analysis platform of single compound regulated kinase spectrum based on the existing big data of kinase activity and the deep neural network algorithm. Based on this method, the average ROC of this method is as high as 0.75 , and the accuracy is significantly higher than other prediction methods [28-34]. Therefore, we used the KinomeX to predict the potential protein kinase targets of 40 important components in CDDP, and obtained 288 kinase targets (Table S4). And then we took this result to filter the 148 targets from above, and obtained the potential direct target set of CDDP for further verification. 37 kinase targets were selected from targets recorded in the databases (Table S5), and 92 kinase targets were selected from Multi-voting SEA predicted results (Table S6). These two parts shared 20 kinase targets (Figure 3), while 109 kinase targets deserve to be verified in total (Table 3, Table S7).

Table 3. Statistics of the number of potential direct kinase targets of CDDP to be verified

\begin{tabular}{cccc}
\hline Target sources & $\begin{array}{c}\text { Number of } \\
\text { targets }\end{array}$ & $\begin{array}{c}\text { Number of kinase } \\
\text { targets }\end{array}$ & $\begin{array}{c}\text { Targets screened by } \\
\text { KinomeX }\end{array}$ \\
\hline Recorded targets & 262 & 55 & 37 \\
Multi-Voting SEA & 377 & 121 & 92 \\
predicted targets & & & 20 \\
Common targets & 160 & 28 & 109 \\
Total targets & 479 & 148 & \\
\hline
\end{tabular}


Kinase targets recorded in database screened by KinomeX

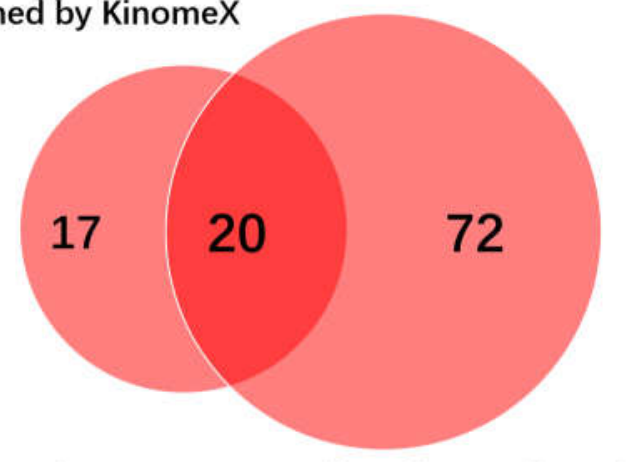

Kinase targets predicted by Multi-Voting SEA screened by KinomeX

Figure 3. The number of potential direct kinase targets of CDDP to be verified

\section{Active kinase targets of CDDP}

Among the above 109 kinase targets, 106 kinase targets were contained in the commercial kinase spectrum developed by Eurofins Company. We test the activity of 106 kinase targets at $25 \mu \mathrm{g} / \mathrm{ml}$ concentration of CDDP. The active targets results were screened according to the threshold described in the method (Table 4).

In total, 30 active targets were obtained, the accuracy was about $28.3 \%$ (Tables 4 and 5, Table S7). Among them, 15 out of 37 recorded kinase targets were verified, and the accuracy is about $40.5 \%$ (Table 4, Table S5). 26 out of 89 kinase targets predicted from Multi-voting SEA got active value, bringing the accuracy up to 29.2\% (Table 4, Table S6). 11 from common 20 kinase targets were verified, achieving 55\% accuracy.

Table 4. Statistical analysis on accuracy of activity test data for the potential direct kinase targets of CDDP

\begin{tabular}{ccccc}
\hline Target sources & $\begin{array}{c}\text { Targets } \\
\text { screened by } \\
\text { KinomeX }\end{array}$ & In Full KP & $\begin{array}{c}\text { Active in } \\
\text { Full KP }\end{array}$ & $\begin{array}{c}\text { Accuracy } \\
\text { (\%) }\end{array}$ \\
\hline $\begin{array}{c}\text { Recorded targets } \\
\text { Multi-Voting SEA predicted }\end{array}$ & 37 & 37 & 15 & 40.5 \\
$\quad$ targets & 92 & 89 & 26 & 29.2 \\
Common targets & 20 & 20 & 11 & 55 \\
Total targets & 109 & 106 & 30 & 28.3 \\
\hline
\end{tabular}


Table 5. Kinase target verified by activity test at $25 \mu \mathrm{g} / \mathrm{ml}$ concentration of CDDP

\begin{tabular}{|c|c|c|c|c|c|}
\hline \# & Kinase & Protein & Gene & Gene ID & $25 \mu \mathrm{g} /$ ml-Activity \%* \\
\hline 1 & Aurora-A(h) & Aurora-A & AURKA & 6790 & 76 \\
\hline 2 & Aurora-B(h) & Aurora-B & AURKB & 9212 & 56 \\
\hline 3 & $\operatorname{Axl}(\mathrm{h})$ & AXL & AXL & 558 & 73 \\
\hline 4 & CaMKII $\gamma(\mathrm{h})$ & CaMK2gamma & CAMK2G & 818 & 64 \\
\hline 5 & CLK1(h) & CLK1 & CLK1 & 1195 & 4 \\
\hline 6 & $\operatorname{Fms}(\mathrm{h})$ & Fms & CSF1R & 1436 & 59 \\
\hline 7 & CK $1 \gamma 3(\mathrm{~h})$ & CK1gamma3 & CSNK1G3 & 1456 & 58 \\
\hline 8 & $\mathrm{CK} 2 \alpha 1(\mathrm{~h})$ & CK2alpha1 & CSNK2A1 & 1457 & 65 \\
\hline 9 & $\mathrm{CK} 2 \alpha 2(\mathrm{~h})$ & CK2alpha2 & CSNK2A2 & 1459 & 64 \\
\hline 10 & DYRK1A(h) & DYRK1A & DYRK1A & 1859 & 80 \\
\hline 11 & FGFR1(h) & FGFR & FGFR1 & 2260 & 69 \\
\hline 12 & Flt1(h) & Flt-1 & FLT1 & 2321 & 78 \\
\hline 13 & Flt3(h) & FLT3 & FLT3 & 2322 & 74 \\
\hline 14 & Fyn(h) & Fyn & FYN & 2534 & 63 \\
\hline 15 & GSK3 $\beta(h)$ & GSK3beta & GSK3B & 2932 & 56 \\
\hline 16 & HIPK4(h) & HIPK4 & HIPK4 & 147746 & 71 \\
\hline 17 & IRAK4(h) & IRAK4 & IRAK4 & 51135 & 80 \\
\hline 18 & $\operatorname{Lck}(\mathrm{h})$ & Lck & LCK & 3932 & 79 \\
\hline 19 & MAPK1(h) & MAPK1(ERK2) & MAPK1 & 5594 & 78 \\
\hline 20 & $\operatorname{Met}(\mathrm{h})$ & Met & MET & 4233 & 43 \\
\hline 21 & $\operatorname{TrkC(h)}$ & TRKC & NTRK3 & 4916 & 72 \\
\hline 22 & $\operatorname{PDGFR} \alpha(\mathrm{h})$ & PDGFRalpha & PDGFRA & 5156 & 76 \\
\hline 23 & Pim-1(h) & Pim1 & PIM1 & 5292 & 69 \\
\hline 24 & Pim-2(h) & Pim2 & PIM2 & 11040 & 71 \\
\hline 25 & $\operatorname{Ret}(\mathrm{h})$ & Ret & RET & 5979 & 61 \\
\hline 26 & Rsk2(h) & RSK2 & RPS6KA3 & 6197 & 78 \\
\hline 27 & $\operatorname{SLK}(\mathrm{h})$ & SLK & SLK & 9748 & 75 \\
\hline 28 & SRPK1(h) & SRPK1 & SRPK1 & 6732 & 74 \\
\hline 29 & DRAK1(h) & DRAK1 & STK17A & 9263 & 79 \\
\hline 30 & $\operatorname{Syk}(\mathrm{h})$ & Syk & SYK & 6850 & 63 \\
\hline
\end{tabular}

* The lower the value, the stronger the binding activity. 
Among them, 14 targets with active value lower than 70 were repeatedly verified at the concentration of $250 \mu \mathrm{g} / \mathrm{ml}$, from which 9 targets with dose-dependent relationship were found, such as MET, PIM1 and SYK (Table 6).

Table 6. Comparison of the kinase targets verified by activity test at $25 \mu \mathrm{g} / \mathrm{ml}$ and $250 \mu \mathrm{g} / \mathrm{ml}$ of CDDP separately

\begin{tabular}{|c|c|c|c|c|c|c|}
\hline$\#$ & Kinase & Protein & Gene & Gene ID & $25 \mu \mathrm{g} / \mathrm{ml}$-Activity \% ${ }^{*}$ & $250 \mu \mathrm{g} / \mathrm{ml}$-Activity \% \\
\hline 1 & Aurora-B(h) & Aurora-B & AURKB & 9212 & 56 & 28 \\
\hline 2 & CaMKII $\gamma(\mathrm{h})$ & CaMK2gamma & CAMK2G & 818 & 64 & 86 \\
\hline 3 & CK $1 \gamma 3(h)$ & CK1gamma3 & CSNK1G3 & 1456 & 58 & 23 \\
\hline 4 & CK2 $2 \alpha 1(h)$ & CK2alpha1 & CSNK2A1 & 1457 & 65 & 47 \\
\hline 5 & CK2 $2 \alpha 2(h)$ & CK2alpha2 & $\mathrm{CSNK} 2 \mathrm{~A} 2$ & 1459 & 64 & 43 \\
\hline 6 & CLK1(h) & CLK1 & CLK1 & 1195 & 4 & 4 \\
\hline 7 & FGFR1(h) & FGFR & FGFR1 & 2260 & 69 & 43 \\
\hline 8 & Fms(h) & Fms & CSF1R & 1436 & 59 & 58 \\
\hline 9 & Fyn(h) & Fyn & FYN & 2534 & 63 & 63 \\
\hline 10 & GSK3 $\beta(h)$ & GSK3beta & GSK3B & 2932 & 56 & 39 \\
\hline 11 & $\operatorname{Met}(\mathrm{h})$ & Met & MET & 4233 & 43 & 8 \\
\hline 12 & Pim-1(h) & Pim1 & PIM1 & 5292 & 69 & 18 \\
\hline 13 & $\operatorname{Ret}(\mathrm{h})$ & Ret & RET & 5979 & 61 & 70 \\
\hline 14 & Syk(h) & Syk & SYK & 6850 & 63 & 2 \\
\hline
\end{tabular}

* The lower the value, the stronger the binding activity. 


\section{Discussion}

Aiming at the problems existing in the research of TCM prescriptions, this study considers that the key to solve the problem is to carry out the research on the direct targets of TCM prescriptions. In this study, we proposed a strategy based on recorded data with definite activity and algorithm prediction data to find the direct kinases target of TCM, which is independent of any specific disease model, and take CDDP as a case study. The results showed that we can quickly obtain the potential direct kinase targets at a lower cost and high reliability with high success rate of verification. The results can lay an important foundation for the study of the mechanism and material basis of TCM from the origin [10], expanding TCM potential new indications and promoting TCM modernization.

TCM prescriptions are the characteristics of TCM. These prescriptions embodies the dialectical thought of TCM and the medication holistic view. The ingredients contained in the prescriptions could be significantly different from those in the single herb. Therefore, in this study, the components contained in CDDP instead of those in single herb were taken as the research objects. As accumulating evidences have proved that the ingredients entering the blood, main metabolites, bioequivalence components compared to the prescription, and active components reported in literatures contribute more to the effects and mechanisms of TCM [35-37], we raised the hypothesis that the potential targets of all the important components mentioned above should be more likely to become the direct targets of the whole prescriptions. In addition, we included another component reported the most in single herbs but has not been confirmed in the whole prescription, quercetin as important component to finalize the list. This method undoubtedly improves the credibility of the data, which is different from most commonly used network pharmacology research flowchart [38-40]. In addition, in order to obtain the potential target data for the important components in the whole recipe, we integrated the recorded data and predicted data. On one hand, the existing research results have been fully utilized by comprehensively collecting the public activity data. On the other hand, in order to avoid missing some important targets, the 
algorithm model of target prediction based on structural similarity was used to predict the potential targets of components. Moreover, kinase targets predicted by KinomeX platform were used to filter the kinase targets obtained by the above two methods, which can further improve the success rate of further verification.

At present, there is no research on the direct target for any compound TCM as a whole. Most of the study focused on active components in TCM. In this study, potential targets of the important components in the complex system of TCM were taken as the start to carry out the direct targets of recipe in vitro. Among them, small molecule affinity chromatography and activity-based protein profiling (ABPP) are the most widely used target identification technologies for active ingredients extracted from Chinese herbal and other natural products. Target fishing technology is a widely used method, which is based on small molecule affinity chromatography [41] and the principle that drug molecules can covalently bind to proteins. The drug molecules are connected to biocompatible inert resin (magnetic beads or agarose gel) through chemical reaction. The target protein of the drug molecule was determined by electrophoresis, purification and high resolution mass spectrometry [42-46]. Using this strategy, a series of targets for active components of TCM have been successfully identified. For example, Tu research group constructed probe for sumitone [47] and chrysanthema lactone [48], successively identified the target of sumitone a as inosine monophosphate dehydrogenase 2 (IMPDH2), and the target of chrysanthema lactone with anti-inflammatory effect was heat shock protein 70 (HSP70). All of them revealed the mechanism of action for active components in TCM from the origin. The target fishing strategy is a highly maneuverable target identification technology, which effectively promotes the clarification for mechanism and speeds up the modernization process of TCM. In the process of using this strategy, if we need to introduce affinity tags with larger steric hindrance, the activity of the compounds may be reduced or even lost. Target fishing technology is suits more for further in-depth analysis to screen the specific ingredients binding individual targets.

14 targets with active value lower than 70 at the concentration of $25 \mu \mathrm{g} / \mathrm{ml}$ were 
verified again at the concentration of $250 \mu \mathrm{g} / \mathrm{ml}$, from which 9 targets with dosedependent relationship were found, such as MET, PIM1 and SYK. However, the activity of four kinases, CAMK2G, CSF1R, FYN and RET, increased. The possible reasons are as follows: firstly, the components with high molecular weight in TCM form great stereo-hindrance effect when the concentration increases, which hinders the combination between active molecules and targets. Secondly, due to the existence of positive effectors in CDDP, when the concentration increases, it produces a positive synergy through the allosteric effect, increasing the protein activity and relatively weakening the inhibition of the components on the activity of targets, such as the synergy weakens the affinity and internal effectiveness of the ligand on the receptors [49-53]. For example, the two components, CDDP 12 (rosmarinic acid) and CDDP 37 (catechol), contained in CDDP can act on the common target FYN. However, the binding site for the two components may be different, which may bring the allosteric effect, weakening the inhibition intensity under the condition of high concentration. These components are not directly binds to protein active sites, but the allosteric sites, outside the active sites of the protein, causing the conformational change of proteins and their activity. In the complex system of TCM, it may be due to the existence of allosteric effectors that the prescriptions can regulate the whole body in a systematic way.

In this study, 106 kinase targets were tested, and finally 30 active targets were obtained, with an accuracy of $28.3 \%$. Among them, 15 out of 37 recorded kinase targets were verified, and the accuracy is about $40.5 \%$. 26 out of 89 kinase targets predicted from Multi-voting SEA got active value, and the accuracy is about $29.2 \%$. The 11 in the common 20 kinase targets were verified, the accuracy was 55\%. As expected, the success rate of the known kinase targets is higher than that of the predicted targets. Hoverer, the candidate targets for verification is toolimited, and some key targets may be missed. By contrary, the targets obtained by predictive method can greatly expand the number of targets to be verified. The filter by the KinomeX predictive results enables a higher success rate at a lower cost. For any component of TCM prescription 
or western medicine, we can efficiently obtain the kinase targets to be verified through this system.

According to the current research results, the following related research can be carried out in the future: Firstly, we can predict and verify the specific components or component group in CDDP potentially regulating the active kinase targets obtained in this research. Thereby, the material basis of TCM can be elaborate. Secondly, we can use the active kinase targets to elucidate the mechanism of action for CDDP from a brand-new perspective. Thirdly, we can further compare and analyze the feature genes of some diseases and target genes modulated by CDDP, providing informative rationales for CDDP repositioning in the future.

The composition of CDDP is relatively simple, which only includs Radix Salviae (Danshen), Panax Notoginseng (Burk.) F. H. Chen Ex C. Chow (Sanqi), Borneolum Syntheticum (Bingpian). If we use the strategy mentioned above to screen direct targets for other complex prescriptions in the future, more factors will be taken into account Considering the complexity and diversity of ingredients in prescriptions, a variety of algorithms based on different principles to predict the relationship between components and targets can be used to obtain the potential target data of ingredients [ㄴ-58]. For example, the model predicting drug-target relationship based on network topology parameters [55], based on drug structure similarity and target structure similarity [56], based on clustering multi-dimensional drug target data [57], and based on deep learning and heterogeneous network [됴] etc. By integrating the data predicted by various algorithms, the success rate may be improved. In addition, molecular docking technology can also be used to gain more reliable targets for further verification [59, $\underline{60}]$.

\section{Conclusion}

In this study, 30 direct targets of CDDP were obtained by the strategy mentioned above. This strategy is independent of any specific disease model, and can efficiently obtain the potential direct targets of TCM. Moreover, this method takes the TCM as a whole research object, which is in line with the holistic view and systematic theory of 
TCM, conforming to the guiding principles of pharmacology theory of TCM. The research results of the direct targets of TCM not only provide the theoretical basis for elucidating for the mechanism of action and the material basis, but also indicating rationales for the research of drug repositioning, which is of great significance for promoting TCM modernization. 


\section{Supplemental information}

Table S1 known targets of important ingredients obtained from public database.

Table S2 potential direct targets of important components predicted through Multivoting SEA algorithm.

Table S3 potential direct targets of CDDP.

Table S4 protein kinase targets of important components predicted by KinomeX.

Table S5 37 kinase targets to be verified selected from known targets.

Table S6 92 kinase targets to be verified selected from targets predicted through

Multi-voting SEA algorithm.

Table S7 potential direct kinase targets of CDDP to be verified.

Table S8 statistics of accuracy of activity test results for the target set to be verified without screening by targets obtained from KinomeX.

\section{Acknowledgments}

The authors acknowledge the support provided by students in Professor Lin's Lab.

\section{Declaration of interests}

The authors declare no competing interests. 


\section{References}

1. You-You, T., N. Mu-Yun, Z. Yu-Rong, L. Lan-Na, C. Shu-Lian, Z. Mu-Qun, W. Xiu-Zhen, J. Zheng, and L. Xiao-Tian, Studies on the constituents of Artemisia annua Part II. Planta Med, 1982. 44(3): p. 143-5.

2. Chen, X., C. Zheng, C. Wang, Z. Guo, S. Gao, Z. Ning, C. Huang, C. Lu, Y. Fu, D. Guan, A. Lu, and Y. Wang, Systems-Mapping of Herbal Effects on Complex Diseases Using the NetworkPerturbation Signatures. Front Pharmacol, 2018. 9: p. 1174.

3. Zhao, P., J. Li, L. Yang, Y. Li, Y. Tian, and S. Li, Integration of transcriptomics, proteomics, metabolomics and systems pharmacology data to reveal the therapeutic mechanism underlying Chinese herbal Bufei Yishen formula for the treatment of chronic obstructive pulmonary disease. Mol Med Rep, 2018. 17(4): p. 5247-5257.

4. Wu, G.S., H.K. Li, and W.D. Zhang, Metabolomics and its application in the treatment of coronary heart disease with traditional Chinese medicine. Chin J Nat Med, 2019. 17(5): p. 321330.

5. Yang, M. and L. Lao, Emerging Applications of Metabolomics in Traditional Chinese Medicine Treating Hypertension: Biomarkers, Pathways and More. Front Pharmacol, 2019. 10: p. 158.

6. Li, J., P. Zhao, L. Yang, Y. Li, Y. Tian, and S. Li, System biology analysis of long-term effect and mechanism of Bufei Yishen on COPD revealed by system pharmacology and 3-omics profiling. Sci Rep, 2016. 6: p. 25492.

7. Suo, T., H. Wang, and Z. Li, Application of proteomics in research on traditional Chinese medicine. Expert Rev Proteomics, 2016. 13(9): p. 873-81.

8. Wong, V.K., B.Y. Law, X.J. Yao, X. Chen, S.W. Xu, L. Liu, and E.L. Leung, Advanced research technology for discovery of new effective compounds from Chinese herbal medicine and their molecular targets. Pharmacol Res, 2016. 111: p. 546-555.

9. Bao, Y., S. Wang, X. Yang, T. Li, Y. Xia, and X. Meng, Metabolomic study of the intervention effects of Shuihonghuazi Formula, a Traditional Chinese Medicinal formulae, on hepatocellular carcinoma (HCC) rats using performance HPLC/ESI-TOF-MS. J Ethnopharmacol, 2017. 198: p. 468-478.

10. Manville, R.W., J. van der Horst, K.E. Redford, B.B. Katz, T.A. Jepps, and G.W. Abbott, KCNQ5 activation is a unifying molecular mechanism shared by genetically and culturally diverse botanical hypotensive folk medicines. Proc Natl Acad Sci U S A, 2019. 116(42): p. 21236-21245.

11. Liao, W., X. Ma, J. Li, X. Li, Z. Guo, S. Zhou, and H. Sun, A review of the mechanism of action of Dantonic((R)) for the treatment of chronic stable angina. Biomed Pharmacother, 2019. 109: $p$. 690-700.

12. Li, T., Y. Chu, K. Yan, S. Li, X. Wang, Y. Wang, W. Li, X. Ma, J. Yang, and C. Liu, Simultaneous determination of tanshinol, protocatechuic aldehyde, protocatechuic acid, notoginsenoside R1, ginsenoside Rg1 and Rb1 in rat plasma by LC-MS/MS and its application. Biomed Chromatogr, 2017. 31(6): p. 10.1002/bmc.3889.

13. Zhao, L., L. Gaudry, S. Dunkley, T. Brighton, Z.X. Guo, Z.L. Ye, R.Z. Luo, and C.N. Chesterman, Modulation of platelet and leucocyte function by a Chinese herbal formulation as compared with conventional antiplatelet agents. Platelets, 2008. 19(1): p. 24-31.

14. Shuhong, Z., L. Hongjun, C. Bo, X. Li, and S. Bingyin, The Effect of Dantonic Pill on beta-Catenin Expression in a Rat Model of Streptozotocin-Induced Early Stage of Diabetic Nephropathy. J 
Diabetes Res, 2013. 2013: p. 848679.

15. Wu, P., T.E. Nielsen, and M.H. Clausen, FDA-approved small-molecule kinase inhibitors. Trends Pharmacol Sci, 2015. 36(7): p. 422-39.

16. Wang, Y., J. Xiao, T.O. Suzek, J. Zhang, J. Wang, Z. Zhou, L. Han, K. Karapetyan, S. Dracheva, B.A. Shoemaker, E. Bolton, A. Gindulyte, and S.H. Bryant, PubChem's BioAssay Database. Nucleic Acids Res, 2012. 40(Database issue): p. D400-12.

17. Ru, J., P. Li, J. Wang, W. Zhou, B. Li, C. Huang, P. Li, Z. Guo, W. Tao, Y. Yang, X. Xu, Y. Li, Y. Wang, and L. Yang, TCMSP: a database of systems pharmacology for drug discovery from herbal medicines. J Cheminform, 2014. 6: p. 13.

18. Huang, L., D. Xie, Y. Yu, H. Liu, Y. Shi, T. Shi, and C. Wen, TCMID 2.0: a comprehensive resource for TCM. Nucleic Acids Res, 2018. 46(D1): p. D1117-D1120.

19. Wang, J.F., H. Zhou, L.Y. Han, X. Chen, Y.Z. Chen, and Z.W. Cao, Traditional Chinese medicine information database. Clin Pharmacol Ther, 2005. 78(1): p. 92-3.

20. Xu, H.Y., Y.Q. Zhang, Z.M. Liu, T. Chen, C.Y. Lv, S.H. Tang, X.B. Zhang, W. Zhang, Z.Y. Li, R.R. Zhou, H.J. Yang, X.J. Wang, and L.Q. Huang, ETCM: an encyclopaedia of traditional Chinese medicine. Nucleic Acids Res, 2019. 47(D1): p. D976-D982.

21. Li, B., C. Ma, X. Zhao, Z. Hu, T. Du, X. Xu, Z. Wang, and J. Lin, YaTCM: Yet another Traditional Chinese Medicine Database for Drug Discovery. Comput Struct Biotechnol J, 2018. 16: p. 600610.

22. Gaulton, A., A. Hersey, M. Nowotka, A.P. Bento, J. Chambers, D. Mendez, P. Mutowo, F. Atkinson, L.J. Bellis, E. Cibrian-Uhalte, M. Davies, N. Dedman, A. Karlsson, M.P. Magarinos, J.P. Overington, G. Papadatos, I. Smit, and A.R. Leach, The ChEMBL database in 2017. Nucleic Acids Res, 2017. 45(D1): p. D945-D954.

23. Gilson, M.K., T. Liu, M. Baitaluk, G. Nicola, L. Hwang, and J. Chong, BindingDB in 2015: A public database for medicinal chemistry, computational chemistry and systems pharmacology. Nucleic Acids Res, 2016. 44(D1): p. D1045-53.

24. Wang, Z., L. Liang, Z. Yin, and J. Lin, Improving chemical similarity ensemble approach in target prediction. J Cheminform, 2016. 8: p. 20.

25. Li, Z., X. Li, X. Liu, Z. Fu, Z. Xiong, X. Wu, X. Tan, J. Zhao, F. Zhong, X. Wan, X. Luo, K. Chen, H. Jiang, and M. Zheng, KinomeX: $a$ web application for predicting kinome-wide polypharmacology effect of small molecules. Bioinformatics, 2019. 35(24): p. 5354-5356.

26. 蔡少青, 王璇, 尚明英, 徐风, and 刘广学. 中药“显效理论”与药物研发新策略. in 第一届 《药学学报》药学前沿论坛暨 2015 年中国药学会中药与天然药物专业委员会会议. 2015. 中国天津.

27. 徐风，杨东辉，尚明英，王璇，and 蔡少青，中药药效物质的“显效形式”、“叠加作用”和“毒 性分散效应”一一由中药体内代谢研究引发的思考. 世界科学技术-中医药现代化, 2014. 16(04): p. 688-703.

28. Avram, S., A. Bora, L. Halip, and R. Curpan, Modelling Kinase Inhibition Using Highly Confident Data Sets. Journal of Chemical Information \& Modeling: p. acs.jcim.7b00729.

29. Bora, A., S. Avram, I. Ciucanu, M. Raica, and S. Avram, Predictive Models for Fast and Effective Profiling of Kinase Inhibitors. J Chem Inf Model, 2016. 56(5): p. 895-905.

30. Lapins, M. and J.E. Wikberg, Kinome-wide interaction modelling using alignment-based and alignment-independent approaches for kinase description and linear and non-linear data analysis techniques. 11(1): p. 339-0. 
31. Merget, B., S. Turk, S. Eid, F. Rippmann, and S. Fulle, Profiling prediction of kinase inhibitorstowards the virtual assay. Journal of Medicinal Chemistry: p. acs.jmedchem.6b01611.

32. Niijima, S., A. Shiraishi, and Y. Okuno, Dissecting kinase profiling data to predict activity and understand cross-reactivity of kinase inhibitors. J Chem Inf Model, 2012. 52(4): p. 901-12.

33. Schürer, S.C. and S.M. Muskal, Kinome-wide Activity Modeling from Diverse Public High-Quality Data Sets. Journal of Chemical Information \& Modeling. 53(1): p. 27-38.

34. Drug Discovery Maps, a Machine Learning Model That Visualizes and Predicts Kinome-Inhibitor Interaction Landscapes. Journal of Chemical Information and Modeling, 2019.

35. Liu, P., H. Yang, F. Long, H.P. Hao, X. Xu, Y. Liu, X.W. Shi, D.D. Zhang, H.C. Zheng, Q.Y. Wen, W.W. Li, H. Ji, X.J. Jiang, B.L. Zhang, L.W. Qi, and P. Li, Bioactive equivalence of combinatorial components identified in screening of an herbal medicine. Pharm Res, 2014. 31(7): p. 1788-800.

36. Li, W., H. Zhou, Y. Chu, X. Wang, R. Luo, L. Yang, N. Polachi, X. Li, M. Chen, L. Huang, X. Yan, Z. Guo, and H. Sun, Simultaneous determination and pharmacokinetics of danshensu, protocatechuic aldehyde, 4-hydroxy-3-methyloxyphenyl lactic acid and protocatechuic acid in human plasma by LC-MS/MS after oral administration of Compound Danshen Dripping Pills. J Pharm Biomed Anal, 2017. 145: p. 860-864.

37. Li, W., Y. Wu, M. Wan, Y. Chu, X. Wang, S. Li, Z. Liu, X. Chen, N. Polachi, S. Zhou, and H. Sun, Simultaneous determination of three saponins in human plasma after oral administration of compound danshen dripping pills by LC-MS/MS and its application in a pharmacokinetic study. J Pharm Biomed Anal, 2019. 169: p. 254-259.

38. Tao, W., X. Xu, X. Wang, B. Li, Y. Wang, Y. Li, and L. Yang, Network pharmacology-based prediction of the active ingredients and potential targets of Chinese herbal Radix Curcumae formula for application to cardiovascular disease. J Ethnopharmacol, 2013. 145(1): p. 1-10.

39. Wei, S., X. Zhou, M. Niu, H. Zhang, X. Liu, R. Wang, P. Li, H. Li, H. Cai, and Y. Zhao, Network pharmacology exploration reveals the bioactive compounds and molecular mechanisms of LiRu-Kang against hyperplasia of mammary gland. Mol Genet Genomics, 2019. 294(5): p. 11591171.

40. Wan, Y., L. Xu, Z. Liu, M. Yang, X. Jiang, Q. Zhang, and J. Huang, Utilising network pharmacology to explore the underlying mechanism of Wumei Pill in treating pancreatic neoplasms. BMC Complement Altern Med, 2019. 19(1): p. 158.

41. 曾克武, 廖理曦, 万彦军, 姜勇, and 屠鹏飞, 基于靶点“钩钓”策略的肉苁蓉苯乙醇苷药 理靶点鉴定及功效解析. 中草药, 2018. 49(01): p. 173-178.

42. Kong, L.-M., X. Deng, Z.-L. Zuo, H.-D. Sun, Q.-S. Zhao, and Y. Li, Identification and validation of p50 as the cellular target of eriocalyxin B. Oncotarget, 2014. 5(22): p. 11354-11364.

43. Li, D., C. Li, L. Li, S. Chen, L. Wang, Q. Li, X. Wang, X. Lei, and Z. Shen, Natural Product Kongensin $A$ is a Non-Canonical HSP9O Inhibitor that Blocks RIP3-dependent Necroptosis. Cell chemical biology, 2016. 23(2): p. 257-266.

44. Liu, C.-X., Q.-Q. Yin, H.-C. Zhou, Y.-L. Wu, J.-X. Pu, L. Xia, W. Liu, X. Huang, T. Jiang, M.-X. Wu, L.C. He, Y.-X. Zhao, X.-L. Wang, W.-L. Xiao, H.-Z. Chen, Q. Zhao, A.-W. Zhou, L.-S. Wang, H.-D. Sun, and G.-Q. Chen, Adenanthin targets peroxiredoxin I and II to induce differentiation of leukemic cells. Nature chemical biology, 2012. 8(5): p. 486-493.

45. Liu, L., Y. Hua, D. Wang, L. Shan, Y. Zhang, J. Zhu, H. Jin, H. Li, Z. Hu, and W. Zhang, A sesquiterpene lactone from a medicinal herb inhibits proinflammatory activity of TNF- $\alpha$ by inhibiting ubiquitin-conjugating enzyme UbcH5. Chemistry \& biology, 2014. 21(10): p. 1341- 
1350.

46. Dong, T., C. Li, X. Wang, L. Dian, X. Zhang, L. Li, S. Chen, R. Cao, L. Li, N. Huang, S. He, and X. Lei, Ainsliadimer A selectively inhibits IKK $\alpha / B$ by covalently binding a conserved cysteine. Nature communications, 2015. 6: p. 6522.

47. Liao, L.X., X.M. Song, L.C. Wang, H.N. Lv, J.F. Chen, D. Liu, G. Fu, M.B. Zhao, Y. Jiang, K.W. Zeng, and P.F. Tu, Highly selective inhibition of IMPDH2 provides the basis of antineuroinflammation therapy. Proc Natl Acad Sci U S A, 2017. 114(29): p. E5986-E5994.

48. Wang, L.C., L.X. Liao, H.N. Lv, D. Liu, W. Dong, J. Zhu, J.F. Chen, M.L. Shi, G. Fu, X.M. Song, Y. Jiang, K.W. Zeng, and P.F. Tu, Highly Selective Activation of Heat Shock Protein 70 by Allosteric Regulation Provides an Insight into Efficient Neuroinflammation Inhibition. EBioMedicine, 2017. 23: p. 160-172.

49. Kume, H., O. Nishiyama, T. Isoya, Y. Higashimoto, Y. Tohda, and Y. Noda, Involvement of Allosteric Effect and $K(\mathrm{Ca})$ Channels in Crosstalk between $B_{2}$-Adrenergic and Muscarinic $M_{2}$ Receptors in Airway Smooth Muscle. International journal of molecular sciences, 2018. 19(7): p. 1999.

50. Massink, A., T. Amelia, A. Karamychev, and A.P. Ijzerman, Allosteric modulation of $G$ proteincoupled receptors by amiloride and its derivatives. Perspectives for drug discovery? Medicinal research reviews, 2019.

51. Nguyen, T., J.-X. Li, B.F. Thomas, J.L. Wiley, T.P. Kenakin, and Y. Zhang, Allosteric Modulation: An Alternate Approach Targeting the Cannabinoid CB1 Receptor. Medicinal research reviews, 2017. 37(3): p. 441-474.

52. Roskoski, R., Jr., A historical overview of protein kinases and their targeted small molecule inhibitors. Pharmacological research, 2015. 100: p. 1-23.

53. Tan, X., P.A. Osmulski, and M. Gaczynska, Allosteric regulators of the proteasome: potential drugs and a novel approach for drug design. Current medicinal chemistry, 2006. 13(2): p. 155165.

54. Cheng, T., M. Hao, T. Takeda, S.H. Bryant, and Y. Wang, Large-Scale Prediction of Drug-Target Interaction: a Data-Centric Review. The AAPS journal, 2017. 19(5): p. 1264-1275.

55. Lu, Y., Y. Guo, and A. Korhonen, Link prediction in drug-target interactions network using similarity indices. BMC bioinformatics, 2017. 18(1): p. 39-39.

56. Peska, L., K. Buza, and J. Koller, Drug-target interaction prediction: A Bayesian ranking approach. Computer methods and programs in biomedicine, 2017. 152: p. 15-21.

57. Zhang, X., L. Li, M.K. Ng, and S. Zhang, Drug-target interaction prediction by integrating multiview network data. Computational biology and chemistry, 2017. 69: p. 185-193.

58. Zong, N., R.S.N. Wong, and V. Ngo, Tripartite Network-Based Repurposing Method Using Deep Learning to Compute Similarities for Drug-Target Prediction. Methods in molecular biology (Clifton, N.J.), 2019. 1903: p. 317-328.

59. Hadianawala, M., A.D. Mahapatra, J.K. Yadav, and B. Datta, Molecular docking, molecular modeling, and molecular dynamics studies of azaisoflavone as dual COX-2 inhibitors and TP receptor antagonists. Journal of molecular modeling, 2018. 24(3): p. 69-69.

60. Kashaw, S.K., S. Agarwal, M. Mishra, S. Sau, and A.K. Iyer, Molecular Docking Analysis of Caspase-3 Activators as Potential Anticancer Agents. Current computer-aided drug design, 2019. 15(1): p. 55-66. 